\title{
Adaptive I-PD controller Design using Variable Reference Model
}

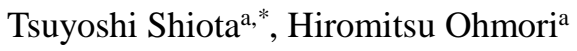 \\ ${ }^{a}$ Keio University, 3-14-1 Hiyoshi, Kohoku-ku, Yokohama, Kanagawa 223-8522, Japan \\ *Corresponding Author: jasdf@z3.keio.jp
}

\begin{abstract}
Model reference adaptive control is one of the useful control schemes which overcome model uncertainty. In conventional adaptive control methods, reference models are designed in advance. However, reference models should be re-designed depending on characteristics of controlled objects. Therefore, in this paper, we propose an adaptive I-PD control system with variable reference model and show the effectiveness by the numerical example.
\end{abstract}

Keywords: Adaptive control, I-PD control, Variable reference model.

\section{Introduction}

Although various control schemes are studied, in the industrial world, the PID control has still accounted for the 80 percent or more ${ }^{(1)}$. As the reason, the structure of PID control is simple and easy to understand intuitively. As parameter tuning method, Ziegler-Nichols method ${ }^{(2)}, \mathrm{C}-\mathrm{H}-\mathrm{R}$ $\operatorname{method}^{(3)}$, partial model matching method ${ }^{(4)}$, and so on are proposed. In partial model matching method, PID parameters and reference model are calculated based upon partial knowledge about control objects. However, in cases where we have limited information about control objects or there are characteristic changes, we cannot get enough controlled performance. To overcome this difficulty, we propose an adaptive control system which changes not only PID parameters but also reference model.

\section{Problem Statement}

Consider a Single-Input/Single-Output system in Fig. 1 described by

$$
y(t)=G(s) u(t)
$$

where $u(t)$ and $y(t)$ are control input and control output respectively. The control objective is to design $u(t)$ such that asymptotically $y(t)$ tracks $y_{M}(t)$ given by following equation with all generated signals remaining bounded.

$$
y_{M}(t)=G_{M}(s) r(t)
$$

\section{Partial Model Matching Method}

\subsection{Denominator Series Expression}

In partial model matching method by Kitamori, transfer functions of controlled objects are denoted by denominator series expression.

$$
\begin{aligned}
G(s) & =\frac{B(s)}{A(s)} \\
& =\frac{b_{0}+b_{1} s+b_{2} s^{2}+b_{3} s^{3}+\cdots}{a_{0}+a_{1} s+a_{2} s^{2}+a_{3} s^{3}+\cdots} \\
& =\frac{1}{h_{0}+h_{1} s+h_{2} s^{2}+h_{3} s^{3}+\cdots} \\
& =\frac{1}{H(s)}
\end{aligned}
$$

Reference models are similarly given by denominator series expression.

$$
\begin{aligned}
G_{M}(s) & =\frac{1}{1+\sigma s+\alpha_{2}(\sigma s)^{2}+\alpha_{3}(\sigma s)^{3}+\cdots} \\
& =\frac{1}{H_{M}(s)}
\end{aligned}
$$




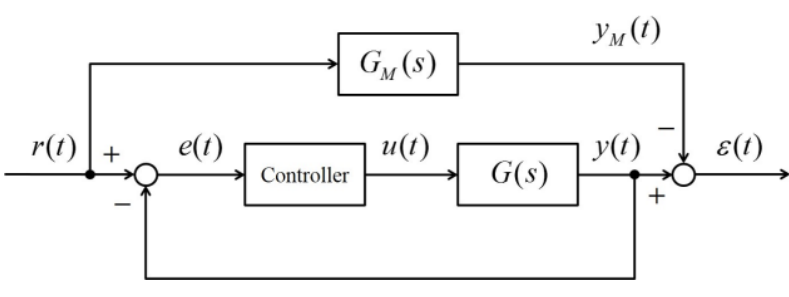

Fig. 1. Control system.

where $\sigma$ is a time-scaling parameter and $\alpha_{i}$ are parameters to adjust the damping characteristics of designed system.

\subsection{Controller Design}

I-PD Controller based on partial model matching method in

Fig. 2, parameters $k, f_{0}, f_{1}$ and $\sigma$ are calculated as

$$
\begin{aligned}
& \sigma=\frac{\alpha_{3} h_{3}}{\alpha_{4} h_{2}} \\
& k=\frac{h_{2}}{\alpha_{3} \sigma^{3}} \\
& f_{0}=k \sigma-h_{0} \\
& f_{1}=k \alpha_{2} \sigma^{2}-h_{1}
\end{aligned}
$$

\section{Adaptive I-PD Control System}

The tracking error $\varepsilon(t)$ is expressed as

$$
\varepsilon(t)=\frac{l s}{H_{M}(s)}\left[u(t)-\theta^{\mathrm{T}} \zeta(t)\right]+v(t)
$$

where

$$
\begin{aligned}
& l=\frac{1}{k} \\
& \theta=\left[\begin{array}{lll}
k & f_{0} & f_{1}
\end{array}\right]^{\mathrm{T}} \\
& \zeta(t)=\left[\begin{array}{lll}
\frac{1}{s} e(t) & -y(t) & -\frac{s}{1+\delta s} y(t)
\end{array}\right]^{\mathrm{T}} \\
& v(t)=-\frac{l s}{H_{M}(s)} \Delta(s) y(t) \\
& \Delta(s)=-\frac{\delta s^{2}}{1+\delta s}+h_{4} s^{4}+h_{5} s^{5}+\cdots
\end{aligned}
$$

However, this error transfer function $l_{s} / H_{M}(s)$ is no longer strictly positive real. A famous technique called error augmentation can be used to avoid this difficulty in finding an adaptation law for this error model. The basic idea of the

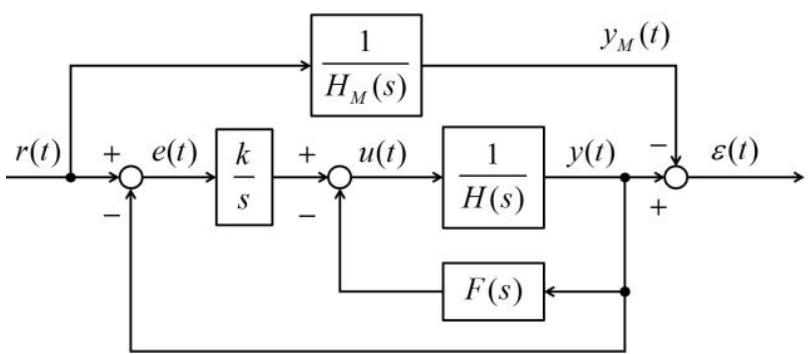

Fig. 2. I-PD control system.

technique is to consider a so-called augmented error $\varepsilon^{\prime}(t)$ which correlates to the parameter error in a more desirable way than the tracking error $\varepsilon(t)$. The augmented error is computed from an auxiliary error $\eta(t)$.

$$
\begin{aligned}
& \varepsilon^{\prime}(t)=\varepsilon(t)+\hat{l}(t) \eta(t) \\
& \eta(t)=\hat{\theta}^{\mathrm{T}}(t)\left[\frac{s}{H_{M}(s)} \zeta(t)\right]-\frac{s}{H_{M}(s)}\left(\hat{\theta}^{\mathrm{T}}(t) \zeta(t)\right)
\end{aligned}
$$

Using the normalizing signal $N(t)$ and dead zone, the control parameters $\hat{\theta}(t)$ and $\hat{l}(t)$ are updated by

$$
\begin{aligned}
& \dot{\hat{\theta}}(t)=-\Gamma \xi_{N}(t) D\left(\varepsilon^{\prime}{ }_{N}(t)\right) \\
& \dot{\hat{l}}(t)=-\gamma \eta_{N}{ }^{\mathrm{T}}(t) D\left(\varepsilon^{\prime}{ }_{N}(t)\right)
\end{aligned}
$$

where

$$
\begin{aligned}
& \varepsilon^{\prime}{ }_{N}(t)=\varepsilon^{\prime}(t) / N(t) \\
& \eta_{N}(t)=\eta(t) / N(t) \\
& \xi_{N}(t)=\xi(t) / N(t) \\
& \xi(t)=\frac{s}{H_{M}(s)} \zeta(t) \\
& N(t)=\rho+m(t) \\
& m(t)=\left|\frac{1}{s} e(t)\right|+|y(t)|+\frac{1}{s+\kappa}(|u(t)|+|y(t)|) \\
& D\left(\varepsilon^{\prime}{ }_{N}(t)\right)=\left\{\begin{array}{cc}
\varepsilon^{\prime}{ }_{N}(t)-d_{0} & \varepsilon^{\prime}{ }_{N}(t)>d_{0} \\
0 & \left|\varepsilon^{\prime}{ }_{N}(t)\right| \leq d_{0} \\
\varepsilon^{\prime}{ }_{N}(t)+d_{0} & \varepsilon^{\prime}{ }_{N}(t)<d_{0}
\end{array}\right.
\end{aligned}
$$

\section{Variable Reference Model}

Using asymptotic stabile polynomial, we can get parametric form as

$$
y(t)=\phi^{\mathrm{T}} \zeta_{I}(t)-w(t)
$$

where 


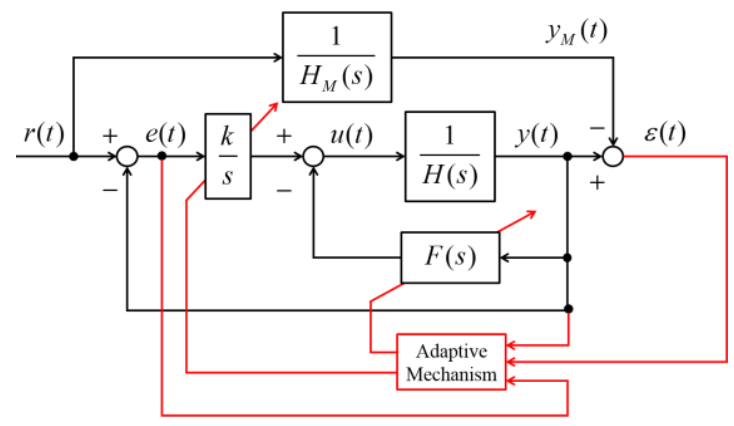

Fig. 3. Adative I-PD control system.

$$
\begin{aligned}
& \phi=\left[\begin{array}{lllll}
1 & \phi_{0} & \phi_{1} & \phi_{2} & \phi_{3}
\end{array}\right]^{\mathrm{T}} \\
& \zeta_{I}(t)=\left[\frac{1}{(s+\lambda)^{3}} u(t) \frac{1}{(s+\lambda)^{3}} y(t)\right. \\
& \left.\frac{s}{(s+\lambda)^{3}} y(t) \quad \frac{s^{2}}{(s+\lambda)^{3}} y(t) \frac{s^{3}}{(s+\lambda)^{3}} y(t)\right]^{\mathrm{T}} \\
& w(t)=\frac{h_{4} s^{4}+h_{5} s^{5}+\cdots}{(s+\lambda)^{3}} y(t)
\end{aligned}
$$

When we define an identification error as

$$
\begin{aligned}
\varepsilon_{I}(t) & =\hat{y}(t)-y(t) \\
& =\phi^{\mathrm{T}} \zeta_{I}(t)+w(t)
\end{aligned}
$$

we can use the following normalized adaptation law.

$$
\dot{\hat{\phi}}(t)=-\Gamma_{I} \zeta_{I_{N}}(t) D\left(\varepsilon_{I_{N}}(t)\right)
$$

where

$$
\begin{aligned}
& \varepsilon_{I_{N}}(t)=\varepsilon_{I}(t) / N_{I}(t) \\
& \zeta_{I_{N}}(t)=\zeta_{I}(t) / N_{I}(t) \\
& N_{I}(t)=\rho_{I}+m_{I}(t) \\
& m_{I}(t)=|y(t)|+\frac{1}{s+\kappa_{I}}(|u(t)|+|y(t)|) \\
& D\left(\varepsilon_{I_{N}}(t)\right)=\left\{\begin{array}{cc}
\varepsilon_{I_{N}}(t)-d_{0} & \varepsilon_{I_{N}}(t)>d_{0_{I}} \\
0 & \left|\varepsilon_{I_{N}}(t)\right| \leq d_{0_{I}} \\
\varepsilon_{I_{N}}(t)+d_{0} & \varepsilon_{I_{N}}(t)<d_{0_{I}}
\end{array}\right.
\end{aligned}
$$

From this identification method, an estimate value of $\hat{\sigma}(t)$ is calculated as

$$
\begin{aligned}
\hat{\sigma}(t) & =\frac{\alpha_{3} \hat{h}_{3}(t)}{\alpha_{4} \hat{h}_{2}(t)} \\
& =\frac{\alpha_{3}\left(\lambda-\hat{\phi}_{3}(t)\right)}{\alpha_{4}\left(3 \lambda^{2}-\hat{\phi}_{2}(t)\right)}
\end{aligned}
$$

\section{Numerical Example}

We consider a following plant:

$$
G(s)=\frac{1}{1+s} e^{-0.5 s}
$$

and a reference model:

$$
\begin{aligned}
& G_{M}(s)=\frac{1}{1+\sigma s+\alpha_{2}(\sigma s)^{2}+\alpha_{3}(\sigma s)^{3}+\alpha_{4}(\sigma s)^{4}} \\
& \left\{\begin{array}{lll}
\alpha_{2} & \alpha_{3} & \alpha_{4}
\end{array}\right\}=\left\{\begin{array}{lll}
\frac{1}{2} & \frac{3}{20} & \frac{3}{100}
\end{array}\right\}
\end{aligned}
$$

Using partial model matching method, the control parameters are calculated as $\sigma=1.1667, k=2.6239$, $f_{0}=2.0612, f_{1}=0.2857$. However, $y(t)$ cannot tracks $y_{M}(t)$ in consequence of modeling error. Hence, we apply adaptive mechanism and re-design $\sigma$ on-line. The control result is shown in Fig. 5, where $\Gamma=1000 I, \gamma=1000$, $\delta=0.001, \rho=0.001, \kappa=0.01, d_{0}=0.0001, \Gamma_{I}=$ $1000 I, \lambda=1, \rho_{I}=0.001, \kappa_{I}=0.1, d_{0_{I}}=0.001$ and the reference signal $r(t)$ is given by a rectangle wave taking values -1 and 1 . From these results, we can confirm asymptotically $y(t)$ tracks $y_{M}(t)$ and adaptive parameters converge as time passes. To evaluate the performance, we compare with Z-N method and CHR method by step responses in Fig. 6. Integral of Absolute value of Error (IAE) and Integral of Squared Error (ISE) is shown in Table 1. 


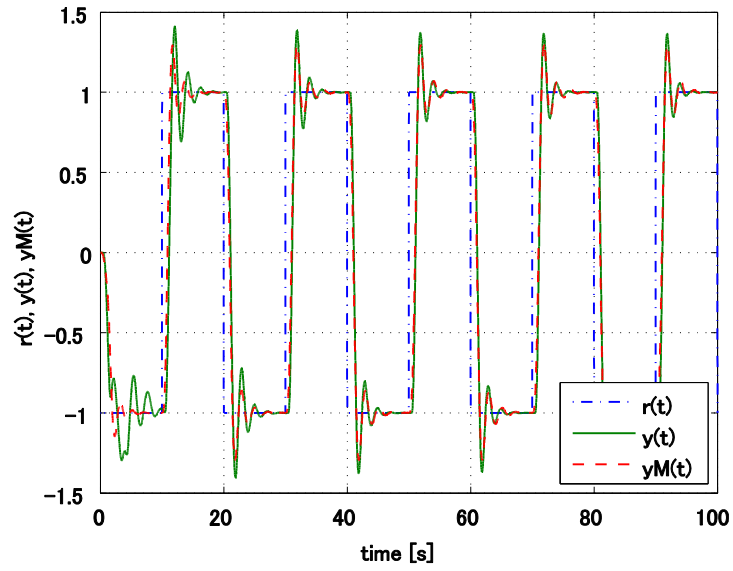

Fig. 5. Control result.

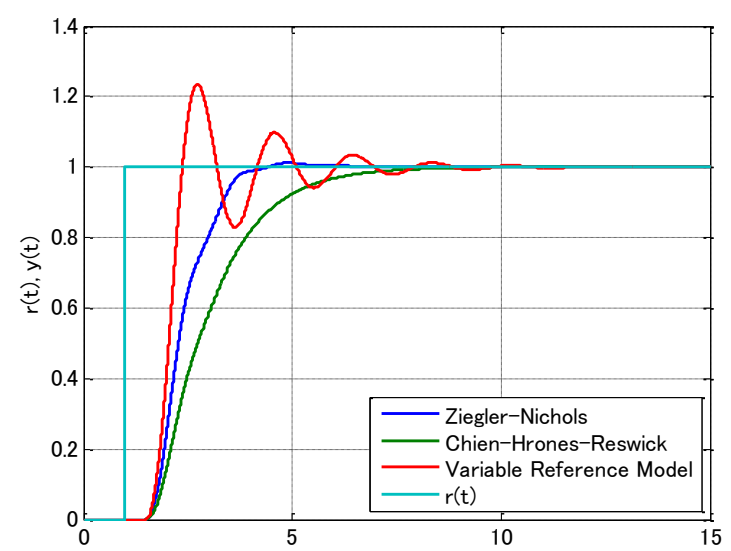

Fig. 6. Step responses.

\section{Conclusions}

In this paper, we propose an adaptive I-PD control scheme with variable reference model. The effectiveness of proposed method is confirmed by comparing controlled performance using IAE and ISE.

\section{References}

(1) M.Kano, and M.Ogawa : "Practice and Challenges in Chemical Process Control Applications in Japan”, Proc. of IFAC World Congress, pp. 10608 - 10613, 2008

(2) J. G. Ziegler, and N. B. Nichols : "Optimum settings for automatic controllers", Trans. ASME, Vol. 64, No. 2, pp. 759-768, 1942

(3) K. L. Chien, J. A. Horones, and J. B. Reswick : "On the Automatic Control of Generalized Passive Systems", Trans. ASME, Vol. 74, pp. 175-185, 1972

(4) T. Kitamori : "A method of control system design
Table 1. Comparison of control performance.

\begin{tabular}{|c|c|c|c|}
\hline & Z-N method & CHR method & $\begin{array}{c}\text { Adaptive } \\
\text { I-PD }\end{array}$ \\
\hline$K_{P}$ & 2.4 & 1.9 & 2.9215 \\
\hline$K_{I}$ & 2.4 & 1.3971 & 4.0369 \\
\hline$K_{D}$ & 0.6 & 0.4465 & 0.3253 \\
\hline IAE & 1.4449 & 2.0758 & 1.3987 \\
\hline ISE & 1.1100 & 1.4392 & 0.9527 \\
\hline
\end{tabular}

based upon partial knowledge about controlled processes", Trans. SISE Japan, Vol. 15, No. 4, pp. 549-555, 1979

(5) T. Shiota and H. Ohmori : "Design of adaptive I-PD control system with variable reference model", Proc. Australian Control Conference, pp. 115-120, 2013 